wissen kompakt $2021 \cdot 15: 101-102$

https://doi.org/10.1007/s11838-021-00132-6

(c) Freier Verband Deutscher Zahnärzte (FVDZ) and Springer Medizin Verlag $\mathrm{GmbH}$, ein Teil von Springer Nature 2021

\section{Autoren}

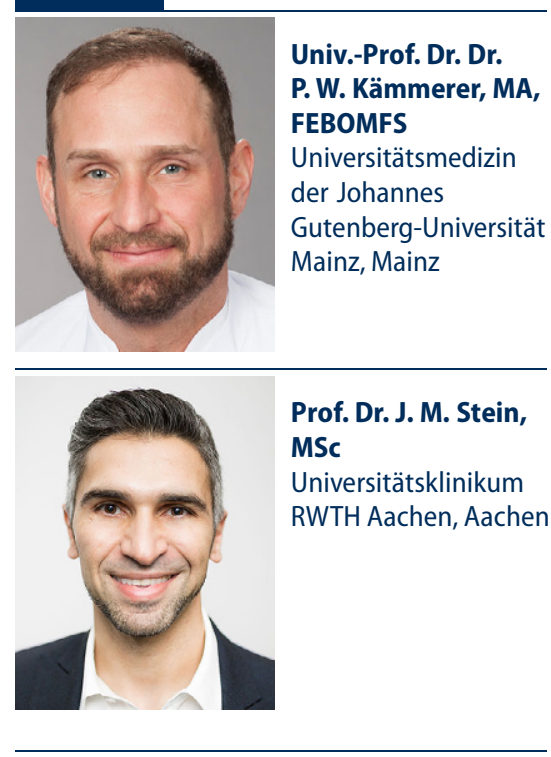

\title{
Neues zu Antibiotika in der Zahnmedizin
}

\author{
P. W. Kämmerer' · J. M. Stein ${ }^{2}$ \\ 'Klinik und Poliklinik für Mund-, Kiefer- und Gesichtschirurgie, plastische Operationen, \\ Universitätsmedizin der Johannes Gutenberg-Universität Mainz, Mainz, Deutschland \\ ${ }^{2}$ Klinik für Zahnerhaltung, Parodontologie und Präventive Zahnheilkunde, Universitätsklinikum RWTH \\ Aachen, Aachen, Deutschland
}

Insgesamt existiert in der Medizin der Trend, weniger Antibiotika zu verschreiben. Dieser Trend ist auch sicherlich den gesteigerten Resistenzentwicklungen geschuldet, also dem weltweiten Zunehmen an gegen Antibiotika resistenten Bakterien. So erhielten nach Angaben der Techniker Krankenkasse (TK) im Jahr 2010 noch ungefähr 30\% der Patienten mit leichten Atemwegserkrankungen Antibiotika, während es 2019 nur noch knapp $15 \%$ waren.

\section{॥ Im Rahmen der ersten Coronawelle kam es sogar zu einem Rückgang der Antibiotikaverordnungen}

Im Rahmen der ersten Coronawelle kam es sogar zu einem Rückgang der Antibiotikaverordnungen von $43 \%$ im Vergleich zum Vorjahr, kausal zum einen auf die in diesem Kontext zurückgegangenen Arztbesuche, aber sicherlich auch indirekt auf die erfolgreichen Maßnahmen zur Eindämmung der Pandemie zurückzuführen.

Während das eine "Lager" die Anwendung von Antibiotika als einen unverzichtbaren und durchaus großzügig einzusetzenden Bestandteil der modernen Medizin sieht, existiert auch ein "Gegenlager", in dem eher die Meinung vorliegt, Antibiotika zögerlich, wenn überhaupt einzusetzen. Gesichert ist auf jeden Fall, dass Antibiotika zur Behandlung bakterieller Infektionen oft die einzigen lebensrettenden Arzneimittel darstellen und dass sie, wie 2016 von dem damaligen Bundesminister für Gesundheit, Hermann Gröhe, betont, eine tragende Säule des Gesundheitsversorgung darstellen. Er fügte allerdings hinzu, dass ihre Wirkung maßgeb- lich davon abhängt, wie wir sie anwenden. Der sachgerechte und indikationsbezogene Gebrauch von Antibiotika ist also der wesentliche und entscheidende Punkt. Somit gilt es, die sachgemäße Antibiotikaanwendung zu verbessern und falsche, unkritisch verordnete Einnahmen zu verhindern. Aber wo ist die wissenschaftliche Evidenz? Für welche Indikationen in der Zahnmedizin sind sie unverzichtbar, und in welchen Fällen ist unter Gabe einer Antibiose ein wirklich besseres Ergebnis im Sinne einer Reduktion von Infektionen und einer akzelerierten Heilung zu erreichen?

Die vorliegende Ausgabe von wissen kompakt haben wir daher gemeinsam mit renommierten Autoren diesem kontroversen und hoch aktuellen Thema gewidmet und hoffen, dass wir wesentliche, für lhre klinische Praxis relevante Indikationsspektren beleuchten konnten. Im ersten Teil dieses Hefts erfahren Sie in zwei Beiträgen mehr über die Gabe von Antibiotika im Rahmen odontogener Infektionen, aber auch im Rahmen der durchaus kritisch zu sehenden Endokarditisprophylaxe. Im zweiten Teil wird ein besonderer Fokus auf den Einsatz von Antibiotika in der Parodontologie gelegt.

\section{॥) Für Antibiotika bei parodontalen/ periimplantären Infektionen werden Indikationsempfehlungen erläutert}

Die erst kürzlich durch die AWMF (Arbeitsgemeinschaft der Wissenschaftlichen Medizinischen Fachgesellschaften e.V.) erstellte deutsche Implementierung der neuen S3-Leitlinien der EFP (European Federation of Periodontology) zur Behandlung der Parodontitis Stadium I-III geben zu diesem - über lange Zeit kontrovers disku- 


\section{Editorial}

tierten - Thema Empfehlungen, aber auch z. T. deutliche Einschränkungen. In zwei Beiträgen zum Einsatz lokaler adjuvanter Therapien in der Parodontitistherapie und zur Anwendung systemischer Antibiotika bei parodontalen und periimplantären Infektionen werden diese Indikationsempfehlungen umfassend erläutert.

Wir freuen uns sehr, Ihnen diese Kompaktzusammenfassung der „Antibiotika in der Zahnmedizin" präsentieren zu dürfen und wünschen Ihnen viel Spaß beim Schmökern!

Mit herzlichen Grüßen

Prof. Dr. Jamal M. Stein, MSc.

Univ.-Prof. Dr. Dr. Peer W. Kämmerer, MA, FEBOMFS

\section{Korrespondenzadresse}

Univ.-Prof. Dr. Dr. P. W. Kämmerer, MA, FEBOMFS

Klinik und Poliklinik für Mund-, Kiefer- und Gesichtschirurgie, plastische Operationen, Universitätsmedizin der Johannes GutenbergUniversität Mainz

Augustusplatz 2, 55131 Mainz, Deutschland peer.kaemmerer@unimedizin-mainz.de

\section{Prof. Dr. J. M. Stein, MSc}

Klinik für Zahnerhaltung, Parodontologie und Präventive Zahnheilkunde, Universitätsklinikum RWTH Aachen

Pauwelsstraße 30, 52074 Aachen, Deutschland jstein@ukaachen.de

Interessenkonflikt. J.M. Stein und P.W. Kämmerer geben an, dass kein Interessenkonflikt besteht. 